\section{ESTUDIO COMPARATIVO DE CLONES DE AISLAMIENTOS DE STAPHYLOCOCCUS AUREUS RESISTENTES A METICILINA PREVALENTES EN LA ARGENTINA}

Los brotes de infecciones nosocomiales causados por cepas de Staphylococcus aureus resistentes a meticilina adquiridas en el hospital (SAMR-HA) han sido consecuencia de la introducción y diseminación de unos pocos clones del microorganismo. Así, en un estudio en el cual se caracterizaron aislamientos de SAMR-HA recuperados de pacientes de dos hospitales de la Universidad de Buenos Aires (1), se detectó una prevalencia de SAMR de $50 \%$ del total de aislamientos de $S$. aureus recuperados y el reemplazo de los clones brasileño (ST239) y pediátrico (ST5) descritos previamente $(2,3)$ por el clon cordobés (ST5).

Desde fines de la década de 1990 se reconoce la emergencia de infecciones en pacientes ambulatorios producidas por cepas de SAMR adquiridas en la comunidad (SAMR-CA) (4). En la Argentina se han identificado al menos cuatro tipos clonales prevalentes (5), de los cuales el que denominamos clon CAA es el más frecuente (48\%); tiene un spa tipo 311, SCCmec IV, leucocidina de Panton Valentine (luk-PV) y pertenece al ST5. Dos casos de meningitis por SAMR-CA en pacientes pediátricos en la comunidad fueron causados por ese tipo clonal (6).

Como la portación de cepas de S. aureus tiene una función muy importante en la epidemiología y patogénesis de las infecciones por ese microorganismo (7), se realizó un estudio prospectivo observacional en una población infantil (8) en el que se encontró que $4,5 \%$ de los individuos estudiados eran portadores de SAMR de dos subtipos diferentes (A1 y A2) del mismo tipo clonal (CAA).

Para conocer los genes de virulencia presentes en los principales clones de SAMR detectados en la Argentina, se seleccionaron tres aislamientos de SAMR-HA de los clones cordobés, brasileño y pediátrico (1-3), tres aislamientos del clon CAA (5) (uno recuperado de infección de piel y partes blandas y dos de bacteriemia) y dos aislamientos obtenidos de niños portadores (8), A1 y A2, en los que se determinó la presencia de genes que codifican para factores de virulencia, entre ellos, enterotoxinas (sea, seb, sec, sed, see, seg, seh, sei, sej), hemolisina (hlg), luk-PV, adhesinas (fnbA, fnbB, clfA, clfB y fib) (9-11), la región ACME- (12), el tipo de agr presente (13) y los implicados en la formación de biopelículas (ica). Los resultados obtenidos se resumen en el cuadro 1.

El clon brasileño (ST239, CC8) tiene una demostrada habilidad para adherirse e invadir las células del epitelio bronquial (14). Coincidentemente, el aislamiento representativo de ese clon fue el único en el que se encontraron todos los genes de adhesinas buscados. En él se detectaron, además, los genes ica, $h l g$, y su $a g r$ fue el único de tipo I, pero mostró sólo uno de los genes codificantes de las enterotoxinas buscados, el sei. Por ser un clon hospitalario clásico, no sorprendió que fuera $l u k$-PV negativo. Del mismo modo, en ninguno de los clones hospitalarios pertenecientes al ST5 (pediátrico y cordobés) se encontraron los genes sea ni $l u k$-PV.

El clon CAA (ST5) y el subtipo A1 de la población de portadores presentaron un patrón de genes de toxinas característico y el mismo perfil de genes de adhesinas, además de los genes ica, hlg y agr de tipo II. En el pasado, la detección de luk-PV se ha asociado con mayor gravedad de los cuadros infecciosos resultantes (15); esta asociación es hoy motivo de debate.

El potencial patogénico de una cepa varía como resultado del intercambio de genes dentro de la especie por transferencia horizontal. Si bien en muchos trabajos se asocia la presencia de determinados factores de virulencia con la mayor capacidad de las cepas de producir infecciones invasivas o de permanecer en sus

CUADRO 1. Caracterización molecular de aislamientos representativos de los clones prevalentes

\begin{tabular}{|c|c|c|c|c|c|c|}
\hline & \multicolumn{6}{|c|}{ Origen/denominación } \\
\hline & \multirow{2}{*}{$\frac{\text { SAMR-CA }}{\text { CAA }}$} & \multicolumn{2}{|c|}{ Portación } & \multicolumn{3}{|c|}{ SAMR-HA } \\
\hline & & $\mathrm{A} 2$ & $\mathrm{~A} 1$ & $\mathrm{Bra}$ & Cord & $\overline{\text { Ped }}$ \\
\hline ST & 5 & 5 & 5 & 239 & 5 & 5 \\
\hline $\mathrm{SCC} m e c$ & IV & IV & IV & III & I & IV \\
\hline$a g r$ & II & II & II & I & II & II \\
\hline ACME & - & - & - & - & - & - \\
\hline sea & + & - & + & - & - & - \\
\hline seb & - & - & - & - & - & - \\
\hline $\mathrm{sec}$ & - & - & - & - & - & - \\
\hline sed & - & - & - & - & - & - \\
\hline see & - & - & - & - & - & - \\
\hline seg & + & + & + & - & - & + \\
\hline seh & - & - & - & - & - & - \\
\hline sei & + & + & + & + & + & + \\
\hline sej & - & - & - & - & - & - \\
\hline hlg & + & + & + & + & + & + \\
\hline luk-PV & + & - & + & - & - & - \\
\hline ica & + & + & + & + & + & + \\
\hline$f n b A$ & + & + & + & + & + & + \\
\hline$f n b B$ & - & - & - & + & - & - \\
\hline$f i b$ & + & + & + & + & + & + \\
\hline clfA & + & + & + & + & + & + \\
\hline$c l f B$ & + & + & + & + & - & + \\
\hline
\end{tabular}

SAMR-HA: Staphylococcus aureus resistente a meticilina adquirido en el hospital SAMR-CA: Staphylococcus aureus resistente a meticilina adquirido en la comunidad.

Bra: clon brasileño; Cord: clon cordobés; Ped: clon pediátrico. 
sitios de colonización, ningún factor de virulencia es, por sí mismo, responsable de la infección.

Nuestros hallazgos muestran la existencia en nuestro medio de un clon perteneciente al linaje ST5, con demostrada plasticidad para adquirir nuevos genes y establecerse en distintos ambientes.

Noella Gardella
Silvina Fernandez
Sabrina Di Gregorio
Arabela Cuirolo
Gabriel Gutkind
Marta Mollerach
Universidad de Buenos Aires
Facultad de Farmacia y Bioquímica
Cátedra de Microbiología
Correspondencia: Noella Gardella, noellag@ffyb.uba.ar

\section{REFERENCIAS}

1. Gardella N, Picasso R, Predari SC, Lasala M, Foccoli M, Benchetrit G, et al. Methicillin-resistant Staphylococcus aureus strains in Buenos Aires teaching hospitals: replacement of the multidrug resistant South American clone by another susceptible to rifampin, minocycline and trimethoprim-sulfamethoxazole. Rev Argent Microbiol. 2005;37(3):156-60.

2. Corso A, Santos Sanches I, Aires de Sousa M, Rossi A, de Lencastre H. Spread of a methicillin-resistant and multiresistant epidemic clone of Staphylococcus aureus in Argentina. Microb Drug Resist. 1998;4(4):277-88.

3. Da Silva Coimbra MV, Teixeira LA, Ramos RL, Predari SC, Castello L, Famiglietti A, et al. Spread of the Brazilian epidemic clone of a multiresistant MRSA in two cities in Argentina. J Med Microbiol. 2000;49(2):187-92.

4. Deurenberg RH, Stobberingh EE. The evolution of Staphylococcus aureus. Infect Genet Evol. 2008;8(6):747-63.

5. Gardella N, von Specht M, Cuirolo A, Rosato A, Gutkind G, Mollerach M. Community-associated methicillin-resistant
Staphylococcus aureus, eastern Argentina. Diagn Microbiol Infect Dis. 2008;62(3):343-7.

6. von Specht M, Gardella N, Tagliaferri P, Gutkind G, Mollerach M. Methicillin-resistant Staphylococcus aureus in communityacquired meningitis. Eur J Clin Microbiol Infect Dis. 2006; 25(4):267-9.

7. Wertheim HF, Vos MC, Ott A, van Belkum A, Voss A, Kluytmans JA, et al. Risk and outcome of nosocomial Staphylococcus aureus bacteraemia in nasal carriers versus non-carriers. Lancet. 2004;364(9435):703-5.

8. Gardella N, Murzicato S, Di Gregorio S, Cuirolo A, Desse J, Crudo F, et al. Prevalence and characterization of methicillinresistant Staphylococcus aureus among healthy children in a city of Argentina. Infect Genet Evol. 2011;11(5):1066-71.

9. Lina G, Piemont $Y$, Godail-Gamot F, Bes M, Peter MO, Gauduchon V, et al. Involvement of Panton-Valentine leukocidin-producing Staphylococcus aureus in primary skin infections and pneumonia. Clin Infect Dis. 1999;29(5):1128-32.

10. Nashev D, Toshkova K, Salasia SI, Hassan AA, Lammler C, Zschock M. Distribution of virulence genes of Staphylococcus aureus isolated from stable nasal carriers. FEMS Microbiol Lett. 2004:233(1):45-52.

11. Tristan A, Ying L, Bes M, Etienne J, Vandenesch F, Lina G. Use of multiplex PCR to identify Staphylococcus aureus adhesins involved in human hematogenous infections. J Clin Microbiol. 2003;41(9):4465-7.

12. Diep BA, Stone GG, Basuino L, Graber CJ, Miller A, des Etages $\mathrm{SA}$, et al. The arginine catabolic mobile element and staphylococcal chromosomal cassette mec linkage: convergence of virulence and resistance in the USA300 clone of methicillinresistant Staphylococcus aureus. J Infect Dis. 2008;197(11): 1523-30.

13. Gilot P, Lina G, Cochard T, Poutrel B. Analysis of the genetic variability of genes encoding the RNA III-activating components Agr and TRAP in a population of Staphylococcus aureus strains isolated from cows with mastitis. J Clin Microbiol. 2002; 40(11):4060-7.

14. Amaral MM, Coelho LR, Flores RP, Souza RR, Silva-Carvalho MC, Teixeira LA, et al. The predominant variant of the Brazilian epidemic clonal complex of methicillin-resistant Staphylococcus aureus has an enhanced ability to produce biofilm and to adhere to and invade airway epithelial cells. J Infect Dis. 2005; 192(5):801-10.

15. Labandeira-Rey M, Couzon F, BoissetS, Brown EL, Bes M, Benito Y, et al. Staphylococcus aureus Panton-Valentine leukocidin causes necrotizing pneumonia. Science. 2007;315(5815):1130-3. 